\section{Revista 2020 \\ Med Vol. 28(1)}

Enero-Junio 2020
-
Editorial Neogranadina

\title{
Caracterización de cardiopatías congénitas en Manizales 2010-2016*
}

\author{
Oscar Alberto Villegas Arenas ${ }^{a}$ Juan Manuel Pérez Agudelo ${ }^{b}$ \\ - Daniela García Rojasc - Omar Armando Gutiérrez Durán ${ }^{d}$ \\ - Juan Gabriel Hurtado Lizarralde e Pablo Felipe Jaramillo Ángel ${ }^{f}$ \\ - Juan José Mora Herrerag - David Ocampo Patiñoh - Alexandra Salazar Riañoi
}

Resumen: Las cardiopatías congénitas son la tercera causa de malformaciones congénitas y una de
las principales responsables de la mortalidad en periodo neonatal en el mundo; se originan a partir
de alteraciones en el desarrollo embrionario. La incidencia es variable; en Colombia, se estima que
afectan del 2 al $3 \%$ de los recién nacidos vivos. En $2015,20,8 \%$ de los niños menores de cinco años
afectados con cardiopatías congénitas fallecieron a causa de estas alteraciones. El presente es un
estudio descriptivo y retrospectivo. Se incluyeron los datos de 252 historias clínicas de pacientes con
diagnóstico de cardiopatía congénita entre los años 2010 y 2016, el $56 \%$ de la población encontrada
fue de sexo femenino. Predominaron las cardiopatías congénitas no cianosantes ( 87 \%). La mayor
prevalencia fue el ductus arterioso permeable ( $35,3 \%$ ). La ecocardiografía Doppler se utilizó en 98,4
$\%$ de los casos; con respecto al manejo, el $47,2 \%$ de los pacientes estuvieron en seguimiento, siendo

* Artículo de investigación

a MD, MsC, profesor titular, Universidad de Manizales. Manizales, Colombia.

Correo electrónico: ovillegas@umanizales.edu.co ORCID: https://orcid.org/000-0001-9198-4081

b MD, MsC, profesor titular Universidad de Manizales. Manizales, Colombia.

Correo electrónico: jperez@umanizales.edu.co ORCID: https://orcid.org/0000-0003-0803-0426

c MD, Universidad de Manizales. Manizales, Colombia.

Correo electrónico: dgar9416@gmail.com ORCID: https://orcid.org/0000-0003-4440-0359

d Autor de correspondencia. MD, Universidad de Manizales. Manizales, Colombia.

Correo electrónico: omar-gd@hotmail.es ORCID: https://orcid.org/0000-0003-40×29-4296

e MD, Universidad de Manizales. Manizales, Colombia.

Correo electrónico: juan.g.hurtado.95@gmail.com ORCID: https://orcid.org/0000-0002-0334-4015

f MD, Universidad de Manizales. Manizales, Colombia.

Correo electrónico: sjaramilloa.10d@gmail.com ORCID: https://orcid.org/0000-0002-4864-4759

g MD, Universidad de Manizales. Manizales, Colombia.

Correo electrónico: moraherreraj@gmail.com ORCID: https://orcid.org/0000-0002-2017-4724

h MD, Universidad de Manizales. Manizales, Colombia.

Correo electrónico: davidocampo04@gmail.com ORCID: https://orcid.org/0000-0002-0377-8104

i MD, Universidad de Manizales. Manizales, Colombia.

Correo electrónico: alexandrasalazri@gmail.com ORCID: https://orcid.org/0000-0002-2657-1632 
este más común que las alternativas médicas o quirúrgicas. En conclusión, el ductus arterioso permeable, la comunicación interventricular y la comunicación interatrial son las cardiopatías no cianosantes más frecuentes en la población estudiada y se asocian a hipertensión pulmonar que ameritan mayor seguimiento. La tetralogía de Fallot fue la cardiopatía congénita cianosante más común.

Palabras clave: cardiopatías congénitas; defectos del tabique interventricular; ecocardiografía Doppler; conducto arterioso permeable (Decs)

Fecha de recepción: 11/10/2019. Fecha de aprobación: 24/03/2020

Disponible en línea: 11/09/2020

Cómo citar: Villegas Arenas OA, Pérez Agudelo JM, García Rojas D, Gutiérrez Durán OA, Hurtado Lizarralde JG, Jaramillo Angel PF, Mora Herrera JJ, Ocampo Patiño D, Salazar Riaño A. Caracterización de cardiopatías congénitas en Manizales 2010-2016. Rev. Med. [Internet]. 11 de septiembre de 2020 [citado 11 de septiembre de 2020];28(1). Disponible en: https://revistas.unimilitar.edu.co/index.php/ rmed/article/view/4313

\section{Characterization of Congenital Heart Defects in Manizales 2010-2016}

Abstract: Congenital heart defects are the third cause of congenital malformations and one of the main causes of mortality in the neonatal period in the world; they originate from alterations in embryonic development. The incidence is variable; in Colombia, it is estimated that they affect 2 to $3 \%$ of live newborns. In 2015, 20.8\% of children under the age of five affected with congenital heart defects died from these disorders. This is a descriptive and retrospective study. Data of 252 clinical records of patients diagnosed with congenital heart defect between 2010 and 2016 were included, showing that $56 \%$ of this population were female. Acyanotic Congenital Heart Diseases predominated (87\%). The highest prevalence was patent ductus arteriosus (35.3\%). Doppler echocardiography was used in $98.4 \%$ of cases; regarding management, $47.2 \%$ of patients were in follow-up, this being more common than medical or surgical alternatives. In conclusion patent ductus arteriosus, ventricular septal defect and interatrial septal defect are the most frequent acyanotic heart diseases in the population under study and they are associated with pulmonary hypertension that require higher follow-up. Tetralogy of Fallot was the most common cyanotic congenital heart disease.

Keywords: congenital heart defects; interventricular septum defects; Doppler echocardiography; patent ductus arteriosus (DeCS)

\section{Caracterização de cardiopatias congênitas em Manizales, Colômbia, 2010-2016}

Resumo: As cardiopatias congênitas são a terceira causa de malformações congênitas e uma das principais responsáveis pela mortalidade no período neonatal no mundo. São originadas a partir de alterações no desenvolvimento embrionário. A incidência é variável; na Colômbia, é estimado que afetam de 2 a 3 \% dos recém-nascidos vivos. Em 2015, 20,8 \% das crianças menores de cinco anos afetadas com cardiopatias congênitas faleceram por causa dessas alterações. Este estudo é descritivo e retrospectivo. Foram incluídos dados de 252 prontuários de pacientes com diagnóstico de cardiopatia congênita entre 2010 e 2016, 56 \% da população encontrada foi do sexo feminino. Predominaram as cardiopatias congênitas não 
cianóticas (87 \%). A maior persistência foi o ducto arterioso permeável (35,3\%). A ecocardiografia Doppler foi utilizada em $98,4 \%$ dos casos; quanto ao manejo, 47,2 \% dos pacientes estiveram em seguimento, o que foi mais comum do que as alternativas médicas ou cirúrgicas. Em conclusão, o ducto arterioso permeável, a comunicação interventricular e a comunicação interatrial são as cardiopatias não cianóticas mais frequentes na população estudada e são associadas com a hipertensão pulmonar que merecem maior seguimento. A tetralogia de Fallot foi a cardiopatia congênita cianótica mais comum.

Palavras-chave: cardiopatias congênitas; defeitos do septo interventricular; ecocardiografia Doppler; ducto arterioso permeável

\section{Introducción}

Anualmente nacen en el mundo 135 millones de niños, de los cuales $3 \%$ presentan enfermedades congénitas; una tercera parte son cardíacas, para un aproximado de 1,3 millones y una prevalencia de 0,5 a 9 casos por cada 1.000 nacidos vivos (1). La prevalencia varía alrededor del mundo: en Suramérica las anomalías cardíacas se presentan en 28 casos por 10.000 nacidos vivos, originando alrededor de 54.000 casos anuales (1); en Colombia, se reporta que las cardiopatías congénitas afectan del 2 al $3 \%$ de los nacidos vivos, constituyendo el 17 $\%$ de todas las anomalías congénitas y siendo el defecto del septo ventricular el de mayor frecuencia, aunque puede existir un subrregistro por aquellas no detectadas al nacimiento (2). En 2015 se reportó que 20,8\% de los niños menores de cinco años con cardiopatías congénitas fallecieron a causa de estas alteraciones. A pesar de que la prevalencia es cercana a 150 por millón de habitantes, solo se intervienen 52 casos por millón (3-5).

Según el informe semestral de defectos congénitos de 2014 realizado por la Dirección Territorial de Salud de Caldas, el 21,5 \% de los recién nacidos (RN) con cardiopatías congénitas fueron diagnosticados prenatalmente y el $75 \%$ fue diagnosticado posnatalmente (6). En un estudio colombiano sobre cardiopatías congénitas realizado en 2016 se tomaron 348 registros del departamento de Caldas; se encontró una frecuencia de 19,8\% para estenosis aórtica, 17,5 $\%$ para comunicación interventricular, 16,1 \% para estenosis pulmonar, $12,9 \%$ para ductus arterioso permeable y 10,6\% para comunicación interatrial (7).

Las cardiopatías congénitas se definen como malformaciones originadas a partir de alteraciones en el desarrollo embrionario entre la tercera y décima semana de gestación; de la etiología multifactorial resultante de la interacción entre aspectos genéticos y ambientales $(7,8)$, se clasifican en cianosantes y no cianosantes (9). Las no cianosantes se dividen en aquellas con flujo pulmonar normal, entre las que se encuentran la estenosis pulmonar, la estenosis aórtica y la coartación aórtica; entre las de flujo pulmonar aumentado están ductus arterioso persistente, la comunicación interatrial y la comunicación interventricular. A su vez, las cianosantes se subdividen en aquellas con flujo pulmonar aumentado como la transposición grandes vasos, el tronco arterioso y el canal atrioventricular; las de flujo pulmonar disminuido engloban la tetralogía de Fallot, como la más frecuente en todas las edades $(7,8,10)$ y la anomalía de Ebstein (11).

La hipertensión pulmonar, caracterizada por un aumento del flujo sanguíneo pulmonar y de las resistencias vasculares, es la complicación más preocupante en países en vía de desarrollo por la dificultad que existe para su diagnóstico y tratamiento. El riesgo de desencadenar hipertensión pulmonar cambia, según la cardiopatía de base, del 10 al $17 \%$ en comunicación interatrial, $50 \%$ en comunicación interventricular y $90 \%$ en defectos completos de tabique atrioventricular. Las ayudas diagnósticas incluyen el ecocardiograma tanto en el periodo fetal como neonatal, el electrocardiograma, la radiografía de tórax y el cateterismo vascular (12).

La ecografía Doppler es el método no invasivo más usado para evaluar la anatomía fetal y detectar las anomalías congénitas, a pesar de que su sensibilidad y especificidad dependen de varios factores como el tipo de malformación, la experiencia del 
examinador, la calidad y tecnología del equipo, entre otras (13). El tratamiento de las cardiopatías congénitas puede ser farmacológico o quirúrgico. El primero se apoya en una amplia variedad de medicamentos, como antiarrítmicos, inotrópicos, diuréticos, vasodilatadores, prostaglandinas, antiprostaglandínicos y otras medidas como la terapia con oxígeno $(12,14)$. Dentro del tratamiento quirúrgico existen técnicas invasivas y mínimamente invasivas; su uso depende del tipo de cardiopatía congénita, el compromiso del paciente, el tamaño del defecto y su respuesta al tratamiento farmacológico $(9,12)$. Este marco general determina la necesidad de establecer, desde la perspectiva de salud pública, la caracterización regional de las cardiopatías congénitas más frecuentes diagnosticadas en el periodo 2010-2016 en Manizales.

\section{Materiales y métodos}

\section{Tipo de estudio}

Estudio cuantitativo, descriptivo y retrospectivo. Se incluyeron datos de pacientes con diagnóstico de cardiopatías congénitas en Manizales (Colombia).

\section{Población y muestra}

La población corresponde a pacientes de tres instituciones de tercer nivel de complejidad de Manizales; la muestra fue de 727 pacientes y sus respectivas historias clínicas, de las cuales 252 cumplieron los criterios de inclusión: diagnóstico establecido de cardiopatía congénita, diagnóstico de menores de edad, paciente vivo e integridad de historia clínica. Para ductus arterioso persistente se excluyeron recién nacidos pretérmino, no fue considerada la altura sobre el nivel del mar como criterio de exclusión. Sí fueron incluidos aquellos que requirieron manejo farmacológico, quirúrgico u observación para los casos en los que persistió por tres días o más como periodo máximo de cierre funcional.

La cardiopatía congénita aislada fue definida como aquella que no tenía otra malformación congénita, la compleja presencia de dos malformaciones cardíacas y polimalformados con tres malformaciones cardíacas o más. No se incluyeron pacientes con síndrome de Down.

\section{Procedimientos e instrumentos}

El trabajo de campo se llevó a cabo entre julio de 2016 y mayo de 2017 e incluyó pacientes diagnosticados en el periodo entre 2010 y 2016. La información se recolectó mediante un instrumento que incluyó variables demográficas y clínicas. Se revisaron las historias clínicas, con orientación a la identificación y determinación de cardiopatías congénitas en los pacientes con edades entre un día y 17 años.

\section{Análisis estadístico}

Se desarrolló análisis bioestadístico para variables categóricas y numéricas. Las variables numéricas se evaluaron con medidas de tendencia central. El chi cuadrado fue el estadígrafo utilizado en el proceso de asociación categórica nominal. Se consideró estadísticamente significativo un valor de $\mathrm{p}$ $<0,05$. El programa utilizado para el análisis fue Statistical Package for Social Sciences (SPSs ${ }^{\circledR}$ ) de la empresa IBM - versión 24.

El estudio fue aprobado por los comités de ética de las instituciones prestadoras de salud en las que se realizó la captura de los datos de las historias clínicas y cumplió con la normatividad legal sobre los aspectos éticos de la investigación en seres humanos para Colombia.

\section{Declaración de aspectos éticos}

La investigación se llevó a cabo con la revisión sistemática de las historias clínicas que fueron provistas por las instituciones en las que se llevó a cabo la investigación; en ninguna parte de la investigación fueron usados ni los nombres ni los documentos de los pacientes partícipes de la investigación. Se firmó un acuerdo de confidencialidad entres las instituciones y todos los participantes de la investigación. De acuerdo con la resolución 8430 de 1993 del Ministerio de Salud de Colombia considerando el capítulo 1 de los aspectos éticos de la investigación en seres humanos que comprende los artículos 5, 7, 8, 10- y según el artículo 11, se clasifica como investigación sin riesgo que no representa amenaza a la autonomía de los seres humanos; no existen riesgos biológicos; contempla 
el principio de beneficencia-no maleficencia; y no incumple los lineamientos de la Declaración de Helsinki de la asamblea médica mundial sobre principios éticos para las investigaciones médicas en seres humanos.

\section{Resultados}

Se incluyeron 252 historias clínicas de pacientes con edades comprendidas entre un día y 17 años. Para unificar la información se analizó la edad por días, obteniéndose una media de 906,3 días, mediana de 60, mínimo de 1 , máximo de 6.453 y desviación estándar de 1.599,4.

El $56 \%$ de los pacientes fueron mujeres y el 44 $\%$ hombres. Los pacientes mayores a un mes de vida (53,6 \%) fueron los más frecuentemente diagnosticados con cardiopatías congénitas respecto a los de edad menor o igual a un mes (46,4 \%). En la distribución general de las cardiopatías congénitas, las formas no cianosantes representaron el $87,3 \%$ de los casos y las de tipo cianosantes 12,7 $\%$ (Tabla 1).

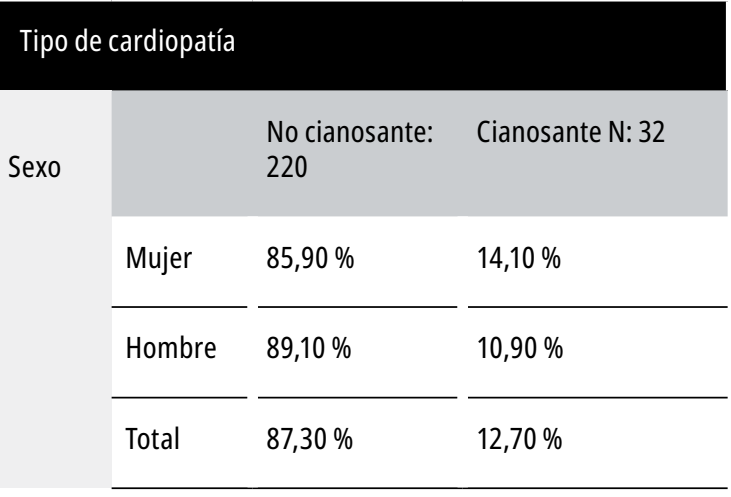

Tabla 1. Distribución tipo de cardiopatía respecto al sexo

Fuente: elaboración propia.

De las cardiopatías no cianosantes predominó el ductus arterioso permeable, seguido de la comunicación interatrial y la comunicación interventricular; en las cardiopatías cianosantes, la más frecuente fue la tetralogía de Fallot y la coartación de la aorta (Figura 1).

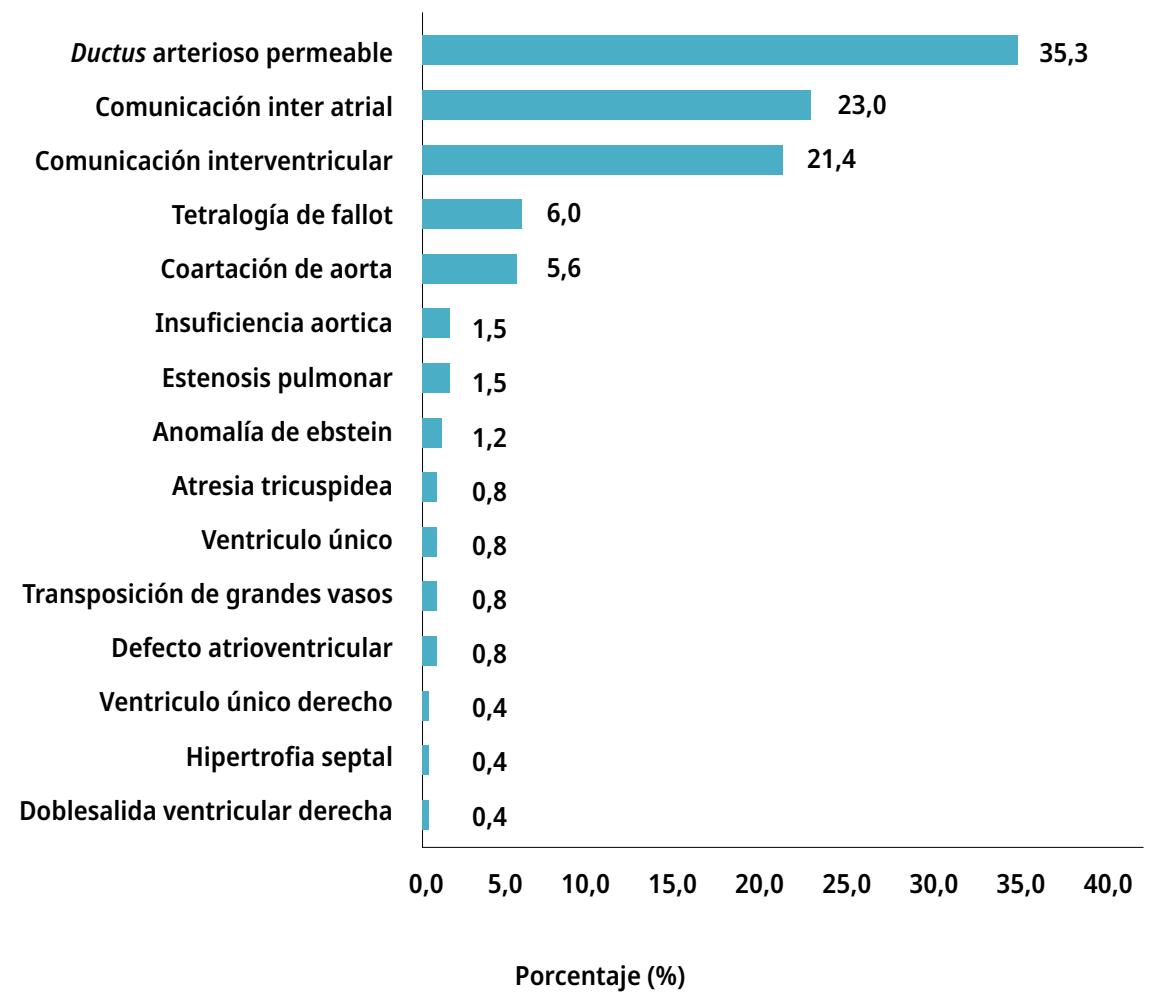

Figura 1. Distribución porcentual específica de las cardiopatías específicas.

Fuente: elaboración propia. 
La cardiopatía más frecuentemente encontrada tanto en hombres como mujeres fue el ductus arterioso permeable; en las mujeres las menos encontradas fueron el ventrículo único y el ventrículo derecho; en los hombres fueron el ventrículo derecho, la hipertrofia septal, la doble salida del ventrículo derecho y el defecto atrioventricular (Tabla 2).

\begin{tabular}{|c|c|c|c|}
\hline & Mujer & Hombre & Total \\
\hline $\begin{array}{l}\text { Ductus arterioso } \\
\text { persistente }\end{array}$ & $35,20 \%$ & $35,50 \%$ & $35,30 \%$ \\
\hline $\begin{array}{l}\text { Comunicación } \\
\text { interatrial }\end{array}$ & $28,90 \%$ & $15,50 \%$ & $23,00 \%$ \\
\hline $\begin{array}{l}\text { Comunicación } \\
\text { interventricular }\end{array}$ & $18,30 \%$ & $25,50 \%$ & $21,40 \%$ \\
\hline Tetralogía de fallot & $5,60 \%$ & $6,40 \%$ & $6,00 \%$ \\
\hline Coartación aórtica & $2,80 \%$ & $9,10 \%$ & $5,60 \%$ \\
\hline $\begin{array}{l}\text { Insuficiencia } \\
\text { aórtica }\end{array}$ & $1,40 \%$ & $1,80 \%$ & $1,60 \%$ \\
\hline Estenosis pulmonar & $0,70 \%$ & $2,70 \%$ & $1,60 \%$ \\
\hline $\begin{array}{l}\text { Anomalía de } \\
\text { Ebstein }\end{array}$ & $2,10 \%$ & $0,00 \%$ & $1,20 \%$ \\
\hline Atresia tricuspídea & $1,40 \%$ & $0,00 \%$ & $0,80 \%$ \\
\hline $\begin{array}{l}\text { Defecto } \\
\text { atrioventricular }\end{array}$ & $0,70 \%$ & $0,90 \%$ & $0,80 \%$ \\
\hline $\begin{array}{l}\text { Transposición de } \\
\text { grandes vasos }\end{array}$ & $1,40 \%$ & $0,00 \%$ & $0,80 \%$ \\
\hline Ventrículo único & $0,70 \%$ & $0,90 \%$ & $0,80 \%$ \\
\hline $\begin{array}{l}\text { Doble salida de } \\
\text { ventrículo derecho }\end{array}$ & 0,00 & 0,90 & 0,40 \\
\hline Hipertrofia septal & 0,00 & 0,90 & 0,40 \\
\hline $\begin{array}{l}\text { Ventrículo único } \\
\text { derecho }\end{array}$ & 0,70 & 0,00 & 0,40 \\
\hline
\end{tabular}

Tabla 2. Distribución porcentual de cardiopatía específica respecto al sexo.

Fuente: elaboración propia.
El $57 \%$ de los pacientes presentaron cardiopatías aisladas, el $37 \%$ presentaron cardiopatías complejas, el $6 \%$ tuvieron polimalformaciones cardíacas, no hubo consideración de pacientes sindrómicos. La asociación más frecuente fue ductus arterioso persistente con comunicación interatrial $(41,1 \%)$ y la segunda más frecuente fue comunicación interatrial con comunicación interventricular (10,3\%).

El 98,4 \% de las cardiopatías congénitas se detectaron por ecocardiografía, seguido por estudios hemodinámicos no especificados $(0,8 \%)$, angiotaC $(0,4 \%)$, cateterismo, arteriografía pulmonar y aortograma torácico (0,4\%). El 47,2\% no recibieron tratamiento, $26,2 \%$ se manejaron con tratamiento farmacológico, $22,6 \%$ con abordaje quirúrgico, y $4 \%$ de los pacientes recibieron manejo mixto (farmacológico y quirúrgico). La comparación de la frecuencia de presentación de cardiopatías frente al proceso de seguimiento mostró $90 \%$ de cumplimiento para ductus arterioso permeable, comunicación interatrial y comunicación interventricular (Tabla 3).

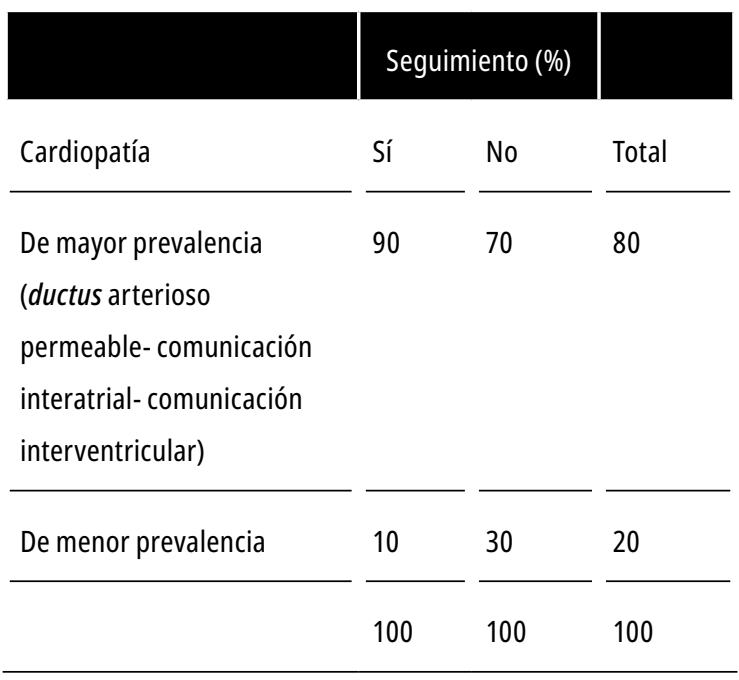

Tabla 3. Comparación entre cardiopatía y seguimiento Fuente: elaboración propia.

\section{Discusión}

Se encontró una mayor prevalencia de cardiopatías congénitas en pacientes de sexo femenino con respecto a sexo masculino; probablemente debido a que los casos en hombres suelen ser más severos 
y fallecen in utero escapando del alcance de este estudio que solo incluyó pacientes vivos. Sin embargo, los datos referentes a dicha distribución varían en las series internacionales; en estudios realizados en Brasil y en Colombia se muestra un predominio masculino (53,4 \% y 51,6 \% respectivamente). Por el contrario, se reporta una prevalencia mayor en mujeres en Alemania (57,3\%) lo que está en concordancia con lo encontrado en la presente investigación $(15,16)$.

En cuanto a la edad de realización del diagnóstico en la población objeto de este estudio, el $53,6 \%$ corresponde a pacientes menores de un año, contrariamente a otros estudios que han reportado que la edad de diagnóstico se presenta por encima del año de vida $(53,4 \%)(16,17)$.

Así mismo, en el presente estudio, la cardiopatía congénita más frecuentemente encontrada fue el ductus arterioso permeable seguido de la comunicación interatrial y la comunicación interventricular; esto discrepa de múltiples series revisadas en las que se describe mayor frecuencia de anomalías como la comunicación interatrial y la comunicación interventricular $(16,19)$. Los casos de ductus arterioso-permeable considerados en este estudio corresponden a casos que requirieron de manejo farmacológico, quirúrgico u observación por ausencia de cierre funcional en el tiempo (20). Debe señalarse que, en el presente estudio, se excluyeron los recién nacidos pretérminos; no fue considerada la altura al nacer como variable de exclusión, pero es importante resaltar que la altura mayor de 2.000 metros sobre el nivel del mar se asocia a una mayor presión de la arteria pulmonar y por consiguiente persistencia del ductus arterioso. En un estudio epidemiológico colombiano realizado por Tassinari y colaboradores., encontraron como principal cardiopatía congénita la comunicación interventricular, discrepando de este estudio que la ubica en tercer lugar (21).

Con respecto a la distribución según el tipo de cardiopatía, hubo predominio de las cardiopatías congénitas no cianosantes. De las cardiopatías congénitas cianosantes la más frecuente fue la tetralogía de Fallot, hallazgo similar al de un estudio realizado en Perú en el cual se encontró un $92 \%$ de cardiopatías no cianosantes y un $8 \%$ de cianosantes y la más frecuente también fue la tetralogía de Fallot (22).

La frecuencia general de cardiopatías congénitas fue mayor en mujeres; un resultado similar se encontró en un estudio en India que reveló asociación significativa entre sexo y defectos cardíacos como la comunicación interventricular (23). En el estudio de Dinaldo de Lima et al. (24) reportan que se diagnosticaron 357 cardiopatías congénitas, el $70,4 \%$ presentaban combinaciones del ductus arterioso permeable con comunicación interatrial, datos similares a los hallazgos de esta investigación.

El ecocardiograma Doppler es el método no invasivo más usado para el diagnóstico y seguimiento de los pacientes con cardiopatías congénitas en la población pediátrica $(13,17,24,25)$, lo cual se correlaciona con los hallazgos de este estudio con un diagnóstico por medio de ecocardiograma Doppler del 98,4\%.

En los reportes ecocardiográficos de la presente serie solo 19,4 \% de los pacientes con cardiopatías congénitas presentaron hipertensión arterial pulmonar pese a lo descrito en la literatura, en la que se reporta como una condición asociada a las principales cardiopatías congénitas $(24,25)$. En nuestra investigación no se presentó tal asociación, lo que puede evidenciar la heterogeneidad en la interpretación de reportes paraclínicos.

En un estudio realizado en el departamento del Cauca (Colombia) entre los años 2003 y 2006 se diagnosticaron 32 pacientes con cardiopatías congénitas, $75 \%$ recibieron tratamiento médico inicial y solo el 25 \% recibió intervención quirúrgica (26); Así mismo, en el artículo de Ana Uribe (22), el $22 \%$ de los pacientes fueron seguidos, el $38 \%$ recibieron tratamiento farmacológico y el 40 $\%$ restante recibieron tratamiento quirúrgico (27). En contraste, en el presente estudio hubo un seguimiento del $47,2 \%$, se trató farmacológicamente al 26,2 \%, quirúrgicamente al 22,6 \% y se realizó manejo combinado al 4,0\%.

\section{Conclusión}

Las cardiopatías congénitas en Manizales se caracterizan por ser mayormente diagnosticadas en los niños mayores a un mes de vida y se presentan con 
mayor frecuencia en las mujeres. Según la clasificación utilizada en las instituciones en las que se realizó el estudio, se encontró que las cardiopatías congénitas más frecuentes pertenecen al grupo de las no cianosantes, con predominio del ductus arterioso persistente, seguido de la comunicación interatrial e interventricular. Las cardiopatías más frecuentes se asocian a hipertensión pulmonar por lo cual ameritan mayor seguimiento. La tetralogía de Fallot fue la cardiopatía congénita cianosante más común. La ecocardiografía sigue siendo la primera línea en el diagnóstico de las cardiopatías congénitas.

\section{Declaración sobre conflictos de interés}

Los autores manifiestan no tener conflictos de interés.

\section{Referencias}

[1] Sandoval N. Congenital Heart Disease in Colombia and Worldwide [Internet]. Rev Colomb Cardiol. 2015;22(1):e1-e2. DoI: https://doi.org/10.1016/j.rccar.2015.03.005

[2] García A, Caicedo M, Moreno K, Sandoval N, Ronderos M, Dennis R. Diferencias regionales en cardiopatías congénitas [Internet]. Rev Colomb Cardiol. 2017;24(2):161-8. DOI: https://doi.org/10.1016/j.rccar.2016.06.012

[3] Liu F, Yang Y, Xie X, Li X, Ma X, Fu Z et al. Prevalence of Congenital Heart Disease in Xinjiang Multi-Ethnic Region of China [Internet]. plos one. 2015;10(8):1-11. DOI: https://doi.org/10.1371/journal.pone.0133961

[4] Knowles RL, Bull C, Wren C, Wade A, Goldstein H, Dezateux C. Modelling Survival and Mortality Risk to 15 Years of Age for a National Cohort of Children with Serious Congenital Heart Defects Diagnosed in Infancy [Internet]. plos one. 2014;9(8).e106896-. DoI: https:// doi.org/10.1371/journal.pone.0106806

[5] Mavroudis CD, Mavroudis C, Jacobs JP, Siegel A. The role of research for sustainable paediatric cardiac programmes in developing countries [Internet]. Cardiol Young. 2012; 22(6):787-95. Dor: https://doi.org/10.1017/ S1047951112001989

[6] Dirección territorial de salud de Caldas. Informe anual defectos congénitos Caldas [Internet]. 2014. Disponible en: http://190.14.226.29/desca/sivigila/Defectos\%20cong\%C3\%A9nitos\%202014.pdf
[7] Madrid AJ, Restrepo JP. Cardiopatías congénitas. Revista Gastrohnup. 2013;15(1):56s72. Disponible en: http://revgastrohnup.univalle.edu.co/a13v15n1s1/ a13v15n1s1art8.pdf

[8] Quesada T, Navarro M. Cardiopatías congénitas hasta la etapa neonatal. Aspectos clínicos y epidemiológicos. Acta Médica del Centro. 2014;8(3):149-162. Disponible en: http://www.revactamedicacentro.sld.cu/index. php/amc/article/view/165/228

[9] Riera C. Actualidades en el tratamiento quirúrgico de las cardiopatías congénitas. Rev Mex Pediatría. 2010;77(5):214-223. Disponible en: https://www.medigraphic.com/pdfs/pediat/sp-2010/sp105g.pdf

[10]Zucker EJ, Koning JL, Lee EY. Cyanotic Congenital Heart Disease Essential Primer for the Practicing Radiologist [Internet]. Radiol Clin n Am. 2017;55(4):693716. DoI: https://doi.org/10.1016/j.rcl.2017.02.009

[11] Sherwin ED, Abrams DJ. Ebstein Anomaly [Internet]. Card Electrophysiol Clin. 2017;9(2):245-54. DoI: https://doi.org/10.1016/j.ccep.2017.02.007

[12]Sun R, Liu M, Lu L, Zheng Y, Zhang P. Congenital Heart Disease: Causes, Diagnosis, Symptoms, and Treatments [Internet]. Cell Biochem Biophys. 2015;72(3):857-60. DoI: https://doi.org/10.1007/s12013015-0551-6

[13] García MA, Imbachí L, Hurtado PM, Gracia G, Zarante I. Detección ecográfica de anomalías congénitas en 76.155 nacimientos en Bogotá y Cali, 2011-2012 [Internet]. Biomédica. 2014;34(3):379-386. DoI: https://doi. org/10.7705/biomedica.v34i3.2259

[14] Rellan S, Garcia C, Paz M. Recién nacido con sospecha de cardiopatía congénita. Protocolos Diagnóstico Terapeúticos de la aep: Neonatología (2008) [Internet]. Disponible en: http://www.enfermeriaaps.com/portal/ download/NEONATOLOGIA/El\%20recien\%20nacido\%20prematuro.pdf

[15] Baltaxe E, Zarante I. Prevalencia de malformaciones cardíacas congénitas en 44,985 nacimientos en Colombia. Arch Cardiol México. 2006;76(3):263-268. Disponible en: http://www.scielo.org.mx/scielo.php?script=sci_arttext\&pid=S1405-99402006000300003

[16] Trevisan P, Diehl Zen T, Machado Rosa R, Nascimiento da Silva J, Bohn Koshiyama D, Paskulin G, et al. Chromosomal Abnormalities in Patients with Congenital Heart Disease [Internet]. Arq Bras Cardiol. 2013;101(6):495-501. DoI: https://doi.org/10.5935/ abc. 20130204

[17] Liu X, Liu G, Wang P, Huang Y, Liu E, Li D, et al. Prevalence of congenital heart disease and its related risk indicators among 90796 Chinese infants aged less 
than 6 months in Tianjin [Internet]. Int J Epidemiol. 2015;44(3):884-893. DoI: https://doi.org/10.1093/ije/ dyv107

[18] Solano L, Aparicio M, Romero JA. Prevalencia e incidencia de cardiopatías congénitas en el Servicio de Cardiología Pediátrica del Hospital Central Militar. Rev Sanid Milit Mex. 2015;69(3):171-178. DoI: https:// doi.org/10.1016/s0300-8932(10)70138-x

[19] García DO, Jiménez ASE, Leal SD, García FY, Ruiz MS. Cardiopatías congénitas diagnosticadas en el Hospital Iván Portuondo en un periodo de 20 años. Panor Cuba Salud. 2012;7(3):14-9.Disponible en: https:/www.medigraphic.com/cgi-bin/new/resumen.cgi?IDARTICU$\mathrm{LO}=49749$

[20]García Guevara C, Schvartz G, Melnik M, García Lugo W, Jakeline Arencibia Faife D, Savío Benavides Carlos García Morejón Cardiocentro Pediátrico A, et al. Sociedad Cubana de Cardiología Ductus arterioso: Ecoanatomía y mecanismos de cierre. CorSalud Abr-Jun. 2015;7(2):135-40. Disponible en: http://www.corsalud. sld.cu/sumario/2015/v7n2a15/ductus.html

[21] Tassinari S, Martínez-Vernaza S, Erazo-Morera N, Pinzón-Arciniegas MC, Gracia G, Zarante I. Epidemiología de las cardiopatías congénitas en Bogotá, Colombia en el período comprendido entre 2001 y 2014 : ¿Mejoría en la vigilancia o aumento en la prevalencia? Biomedica. 2018;38:1-29. DoI: https://doi.org/10.7705/ biomedica.v34i3.2259

[22]Uribe A, Díaz C, Cerrón C. Características epidemiológicas y clínicas de las cardiopatías congénitas en menores de 5 años del Hospital Almanzor Aguinaga Asenjo: Enero - Diciembre 2012 [Internet]. Horiz Méd. 2015;15(1):49-56. DoI: https://doi.org/10.24265/horizmed.2015.v15n1.07

[23]Bhardwaj R, Rai SK, Yadav AK, Lakhotia S, Agrawal D, Kumar A, et al. Epidemiology of Congenital Heart Disease in India [Internet]. Congenit Heart Dis. 2015;10(5):437-446. DoI: https://doi.org/10.1111/ chd. 12220

[24]Leite D, Miziara H, Veloso M. Congenital cardiac malformations in pediatric necropsies: characteristics, associations and prevalence [Internet]. Arq Bras Cardiol. 2010;94(3):294-299. DoI: https://doi.org/10.1590/ S0066-782X2010000300003

[25]Colaco SM, Karande T, Bobhate PR, Jiyani R, Rao SG, Kulkarni S. Neonates with critical congenital heart defects: Impact of fetal diagnosis on immediate and short-term outcomes [Internet]. Ann Pediatr Cardiol. 2017;10(2):126-130. DoI: https://doi.org/10.4103/apc. APC_125_16
[26]Rodríguez VH, Shoemaker RG. Cardiopatías congénitas en niños menores de cinco años. Serie de casos, 2003-2006: Hospital Regional, Santander de Quilichao, Cauca, Colombia. Revista Facultad Ciencias de la Salud. Universidad del Cauca. 2007;9(2):30-35. Dor: https://doi.org/10.18273/revsal.v50n1-2018006

[27]Roth TS, Aboulhosn JA. Pulmonary Hypertension and Congenital Heart Disease [Internet]. Cardiol Clin. 2016;34(3):391-400. DOI: https://doi.org/10.1016/j. ccl.2016.04.002 